EPJ Web of Conferences 116, 03001 (2016)

DOI: $10.1051 /$ epjconf/201611603001

(C) Owned by the authors, published by EDP Sciences, 2016

\title{
A compact array calibrator to study the feasibility of acoustic neutrino detection
}

\author{
M. Ardid ${ }^{a}$, F. Camarena, I. Felis, A. Herrero, C.D. Llorens, J. Martínez-Mora, and M. Saldaña \\ Institut d'Investigació per a la Gestió Integrada de les Zones Costaneres (IGIC) - Universitat \\ Politècnica de València, C/ Paranimf 1, 46730 Gandia, Spain
}

\begin{abstract}
Underwater acoustic detection of ultra-high-energy neutrinos was proposed already in 1950s: when a neutrino interacts with a nucleus in water, the resulting particle cascade produces a pressure pulse that has a bipolar temporal structure and propagates within a flat disk-like volume. A telescope that consists of thousands of acoustic sensors deployed in the deep sea can monitor hundreds of cubic kilometres of water looking for these signals and discriminating them from acoustic noise. To study the feasibility of the technique it is critical to have a calibrator able to mimic the neutrino "signature" that can be operated from a vessel. Due to the axial-symmetry of the signal, their very directive short bipolar shape and the constraints of operating at sea, the development of such a calibrator is very challenging. Once the possibility of using the acoustic parametric technique for this aim was validated with the first compact array calibrator prototype, in this paper we describe the new design for such a calibrator composed of an array of piezo ceramic tube transducers emitting in axial direction.
\end{abstract}

\section{Introduction}

The study of Ultra-High-Energy (UHE) neutrinos by means of the detection of the acoustic pulse generated by the energy deposition after interacting with a nucleus in water was proposed already in 1950s [1]. This phenomenon induces a local heating in a very short period of time leading to a submillisecond pressure pulse signal with bipolar shape and very directive flat disk-like pattern, with the bipolar pulse being emitted perpendicularly to the shower axis direction.

The feasibility of the acoustic detection neutrino technique is currently being studied in the Mediterranean Sea [2,3] and could be implemented in the new deep-sea Cherenkov neutrino telescope under construction, the KM3NeT telescope [4], which will have a volume of several cubic kilometres. Thus, the acoustic detection is a promising technique to cover the detection of UHE neutrinos, being possible to combine these two neutrino detection techniques for a hybrid underwater neutrino telescopes, especially considering that the optical neutrino detection technique needs acoustic sensors for monitoring the position of the optical sensors [5, 6]. A key tool to study the viability of the acoustic detection is an acoustic calibrator able to imitate the bipolar pulse signal. This will be needed to calibrate the sensors, as well as, to improve and train the classification and discernment of the neutrinos bipolar

\footnotetext{
a e-mail: mardid@fis.upv.es
}

This is an Open Access article distributed under the terms of the Creative Commons Attribution License 4.0, which permits unrestricted use, distribution, and reproduction in any medium, provided the original work is properly cited. 
pulse signals from noise or other background transient signals. The proposed design, described in this paper, is a compact calibrator composed of an array of piezo-ceramic tube transducers emitting in axial direction. The emission of the low-frequency acoustic bipolar pulse is generated by parametric emission at high-frequency. For this, specific electronics adapted to the ceramics in order to feed them efficiently are required, as well as, to allow and control the different functionalities the calibrator has to provide.

\section{Compact array calibrator approach}

The use of parametric acoustic sources to generate neutrino-like signals with cylindrical transducers has been achieved and validated in previous studies [7]. The acoustic parametric effect occurs when two intense monochromatic beams with two close frequencies travel together through the medium. Under these conditions, in the region of nonlinear interaction, secondary harmonics of these frequencies are produced at lower frequency with a beam pattern directivity similar to the primary beam. Since this technique offers the advantage of generating a parametric signal much more directive, it allows designing a compact array with fewer units with respect to classical solutions, and thus reducing costs and facilitating the deployment and operation issues. The array calibrator under development will be constituted of a few tube piezo ceramics aligned with optimal distance separation for obtaining an opening angle of about $1^{\circ}$ and phased emission of the multi-element array. The array of transducers will emit at high frequency (hundreds of $\mathrm{kHz}$ ) in the perpendicular direction to the axis and produce a bipolar pulse (with lower frequency spectrum, tens of $\mathrm{kHz}$ ) due to the interaction of parametric signals generated for each element.

The first phase of the calibrator design presented in this paper consists of the study and selection of transducers, their characterization, and the emission of neutrino-like signals with a single transducer using the parametric technique. In a second phase, a study on matching layer materials and transmission loses has to be studied and finalizing the studies of the parametric emission for single and group of transducers, moulding them, designing the electronics and testing them. The last phase will be completing the integration of the system and testing it for long distances. Afterwards, the calibrator will be ready for in situ tests and use either installed in the deep-sea neutrino telescope or from the surface of the sea in a sea campaign with a vessel.

The array calibrator will be able to work in different operation modes; at linear low frequency range by emitting long non-directive signals (easy to detect), at high frequency range by emitting long parametric directive signals, and at high frequency range by emitting the transient and directive parametric bipolar signal. This will allow to use the array transmitter for different functionalities, such as training and tuning the acoustic detector, cross-checking the detector hydrophones, and other marine applications. On the other hand, the different operation modes will allow us to plan a sea campaign in order to facilitate the signal detection in the three steps with increasing difficulty.

\section{Transducer characterization}

Two types of piezo-ceramic tube transducers from UCE ultrasonic Co. LTD have been selected as candidates for the emitters of the array calibrator. The choice was based on resonance frequencies, power emission, dimensions, and costs. Both are able to emit high frequency signals within high power emission and they have a reasonable power level at lower frequencies as well. The first candidate is the piezo-ceramic UCE-534541 with dimensions: outer diameter of $5.3 \mathrm{~cm}$, inner diameter of $4.5 \mathrm{~cm}$ and height of $4.1 \mathrm{~cm}$. The primary resonance frequency is at $490 \mathrm{kHz}$, but it has also a secondary resonance at low frequency, around $35 \mathrm{kHz}$. The second candidate is the piezo-ceramic UCE-343020 with dimensions: outer diameter of $3.4 \mathrm{~cm}$, inner diameter of $3 \mathrm{~cm}$ and height of $2 \mathrm{~cm}$. The primary resonance frequency is at $890 \mathrm{kHz}$, with a secondary resonance frequency around $75 \mathrm{kHz}$. 

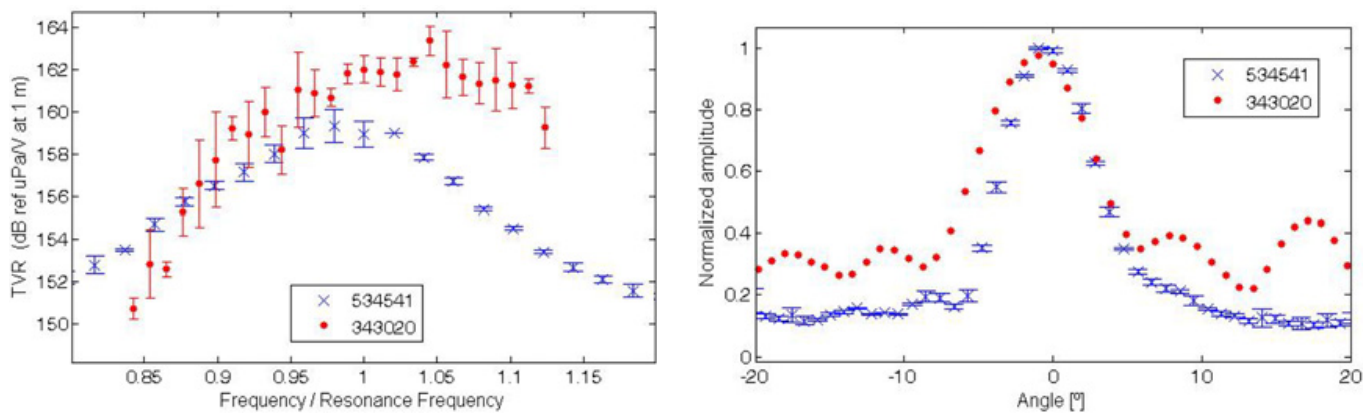

Figure 1. Left: TVR of UCE-534541 at $\mathrm{F}_{\mathrm{R}}=490 \mathrm{kHz}$ (blue $\mathrm{x}$ ) and for UCE-343020 at $\mathrm{F}_{\mathrm{R}}=890 \mathrm{kHz}$ (red dot). Right: Directivity at $490 \mathrm{kHz}$ for the UCE-534541 (blue x) and at $890 \mathrm{kHz}$ for UCE-343020 (red dot).

The piezo-ceramics have been characterized both at high and low resonance frequencies in a water tank of $87.5 \times 113 \times 56.5 \mathrm{~cm}^{3}$ with fresh-water at the laboratory. The calibration of the ceramics has been performed by measuring the Transmitting Voltage Response (TVR) and directivity in emission. The signals used for calibration have been tone bursts with the different frequency ranges of interest of each transducer. The hydrophones used for the calibration are omnidirectional, model RESON-TC40348 for high frequency and model RESON-TC4034 for low frequency. Figure 1 shows the characterization of both ceramics, the left plot shows the sensitivity obtained at high frequency with resonance frequency $\left(F_{R}\right)$ of $490 \mathrm{kHz}$ for the UCE-534541 and $890 \mathrm{kHz}$ for the UCE-343020. The TVR of the UCE-534541 is $159 \mathrm{~dB}(\mathrm{re} \mu \mathrm{Pa} / \mathrm{V}$ at $1 \mathrm{~m})$ at $\mathrm{F}_{\mathrm{R}}(490 \mathrm{kHz})$ with a directivity of $\pm 5^{\circ}$. On the other hand, the TVR of the UCE-343020 is $162 \mathrm{~dB}$ (re $\mu \mathrm{Pa} / \mathrm{V}$ at $1 \mathrm{~m})$ at $\mathrm{F}_{\mathrm{R}}(890 \mathrm{kHz})$ with a directivity of $\pm 7^{\circ}$. The TVR at lower frequencies, $10 \mathrm{kHz}$ to $100 \mathrm{kHz}$, is in the range $132-143 \mathrm{~dB}$ (re $\mu \mathrm{Pa} / \mathrm{V}$ at $1 \mathrm{~m}$ ) for both ceramics [8], so it will be possible to use these ceramics as well for generating low frequency signals directly, which is very useful for calibration purposes.

\section{Parametric bipolar pulse emission}

The experiment of generating the bipolar pulse was carried out in the same water tank. The piezoceramic was connected to a linear $55 \mathrm{~dB}$ RF amplifier ENI 1040L to feed the emitter and generate a more powerful signal in order to achieve the non-linear parametric effect. The chosen receiver was the RESON-TC4034, this transducer is an omnidirectional, broad-band hydrophone with enough sensitivity to detect the primary beam (high frequency) and better sensitivity to lower frequencies for the bipolar pulse detection. Additionally it was connected to a charge amplifier CCA 1000 (Teledyne RESONC) which amplifies the received signal, especially the low frequency range. Here, we present the study performed with the UCE-343020 ceramic, hence, the signal for emission has been derived for parametric generation at $890 \mathrm{kHz}$ as described in ref. [7]. The distance between the emitter and the receiver was $30 \mathrm{~cm}$. The results with the UCE-534541 are reported in ref. [8], where the shapes of the primary beam and the secondary bipolar pulse can be observed in Fig. 3 of this reference.

Since the amplitude of the secondary bipolar signal is about 100 times smaller than the primary beam, it is necessary to use a band pass $([5-80] \mathrm{kHz})$ filter to be able to observe this signal. In order to check that the secondary beam has a non-linear behaviour, the amplitude of the parametric signal, as a function of the voltage applied to the transducer, was measured. Figure 2-left shows the amplitude behaviour of the received signal, without filtering (original) and with the band pass filtering (the parametric bipolar signal, secondary beam). Linear behaviour can be observed for the amplitude of the primary beam, whereas, for the secondary received signal (parametric bipolar signal) the amplitude behaviour is proportional to the square of the amplitude of the input signal, showing the nonlinearity of 

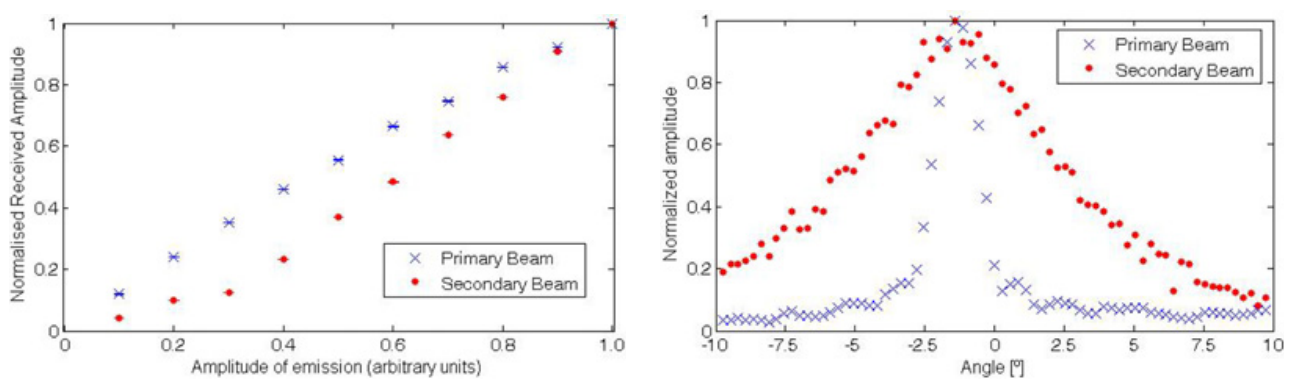

Figure 2. Left: Amplitude of the received primary beam and of the secondary bipolar pulse. Right: Directivity obtained for both signals.

the effect. On the other hand, in Fig. 2-right it can be observed that, despite the frequency content of both signals is so different, the directivity achieved is quite similar.

\section{Conclusions and future steps}

The results obtained on the piezo-ceramics characterization show that both could be good candidates for being part of the future calibrator since they have an optimal sensitivity at both high and low frequency and narrow beam directivity at high frequencies which will lead, in turn, to a very directive low-frequency (parametric) pulse. Moreover, the first studies on the acoustic neutrino-like signals generation by using the parametric technique show that the signal reproduction is achieved.

The next steps will consist in completing the studies of the parametric emission for single and group of transducers, and moulding them. In addition, the electronics design will be finalized and tested. After this, the complete integration of the system and the tests at long distances will be performed before doing sea campaign tests.

We acknowledge the financial support of the Spanish Ministerio de Economía y Competitividad, Grants FPA2012-37528-C02-02, and Consolider MultiDark CSD2009-00064, of the Generalitat Valenciana, Grants ACOMP/2015/175 PrometeoII/2014/079 and of the European FEDER funds.

\section{References}

[1] G.A. Askaryan, J. A. Energy 3, 921 (1957)

[2] J.A. Aguilar, et al., Nucl. Instrum. and Meth. A 626, 128 (2009)

[3] G. Riccobene, Nucl. Instrum. and Meth. A 604, S149 (2009)

[4] The KM3NeT Collaboration, KM3NeT Technical Design Report (2010) ISBN 978-90-6488-0339, available on www. km3net. org

[5] M. Ardid, Nucl. Instrum. and Meth. A 604, S203 (2009)

[6] M. Ardid, Nucl. Instrum. and Meth. A 602, 174 (2009)

[7] M. Ardid et al., Sensors, 12, 4113 (2012), pp. 4113-4132

[8] M. Saldaña et al., New design of an acoustic array calibrator for underwater neutrino telescopes. In Proceedings of the 2nd Int. Electron. Conf. Sens. Appl., 15-30 November 2015; Sciforum Electronic Conference Series, Vol. 2, 2015, E011; doi: 10.3390/ecsa-2-E011 